\title{
New insights of foam flow dynamics in high-complexity 2D
} micromodels

\author{
Christopher Yeates, Souhail Youssef, Elise Lorenceau
}

\begin{abstract}
:
In this work, we present direct observation of foam flow through a 2D porous microfluidic device. Through a specially designed image processing workflow, we perform individual bubble tracking and establish flow dynamics within the micromodel structure. As a comparison to our experimental data, we provide 2D numerical flow simulations intended to serve as a Newtonian reference in a binarized version of the medium, carried out using an in-house Lattice Boltzmann simulation code. The results show that foam flow in our experimental conditions, low gas fraction and high injection velocity, demonstrate a high degree of similarity to the flow of a Newtonian fluid, in aspects of large-scale flow distribution homogeneity and specific flow passage activation. However, the foam data shows a larger spread of porescale flow velocities, spanning from blocked off areas of quasi-zero flow, to zones of high velocity, with velocities reaching values well above the Newtonian counterpart. Another characteristic of the foam flow inside the medium is the dichotomy between the flow patterns of the smallest bubbles, typically dispersing and accessing most regions available, and the largest bubbles, which travel in long straight preferential paths exclusively. We show that intrinsically tied to these different flow patterns is the relationship between bubble velocity and bubble size, as we demonstrate distinct populations of trapped and flowing bubbles with distinct sizes. Finally, we explore the relationships between microstructural parameters and flow intensity and note differences in dependencies in Newtonian and foam cases, while overall showing a generally weak correlation to local structural parameters. Our study, which combines high spatial and time resolution, realistic network dimensions, high network complexity and efficient bubble tracking, therefore sheds new light on the study of foam flow in porous media.
\end{abstract}

\section{Introduction}

Surfactant-stabilized foams are used as injection fluids in porous rocks in Enhanced Oil Recovery (EOR) as a means of improving recovery and improving reservoir conformance of gas injection due to permeability contrast or gravity segregation (Li et al. 2013). In a confined geometry, where the bubble size is often observed to be of the same order as the pore size, foams exhibit a series of original phenomena unseen in either bulk foams such as shear-thinning (Alvarez 2001) or novel properties unknown in other flow situations such as selective mobility control in heterogenous porous media (Kovcek, Bertin, 2003, Tsau 1997 ). Elsewhere in EOR applications, foams can be used to plug areas of a well, to blocking exploited or uninteresting regions. Lastly, we can mention the example of using foams in fractured reservoirs to increase oil recovery in the unfractured rock matrix (Conn et al. 2014; Fernø et al. 2014).

To get a better understanding of foam flow in porous materials, laboratory experiments are often performed either at the core-scale, with observations including apparent viscosity and fluid saturations, or at the pore-scale, through qualitative observation of individual mechanisms or overall oil recovery 
factors usually using transparent 2D micromodels (Ransohoff, Radke 1988; Gauteplass et al. 2015). Although some examples of in-situ flow measurements in porous media are available, various shortcomings may diminish the applicability of these results, such as the unrealistic pore-scale dimensions or low model complexity (low pore-scale heterogeneity or constant coordination number). Despite the lack of direct observation of realistic foam flow, many assumptions are made regarding the precise dynamics of flow based on hypothetical models. For example, Radke, Gillis (1990) describe flowing gas occurring in larger pores whereas intermediate sized pores contain trapped immobile bubbles. Since then, advances in imaging, 2D micromodel fabrication and especially data processing provide new tools to address these complex problems statistically. In particular, how the widths of bubble size and pore distributions interplay with the heterogeneity of the flow field remains an open question. Recently, Géraud et al. (2016), provide interesting results by directly observing these correlations in a 2D medium. In polydisperse foams the authors establish a positive correlation between bubble size and bubble velocity, also observing preferential paths for larger bubbles as well as trapped zones. The author furthermore observes a degree of similarity between the bubble flow maps and the maps relating to pore sizes and affirms that preferential paths occur where large pores are connected together. The authors also reference a structural origin for the existence of trapped zones, yet don't systematically explore this relationship and it remains unclear which structural parameter they allude to. Here we exhaust some of the most obvious candidates including the ones referenced by the authors.

Despite this interesting framework, these open questions suggest that there is still some need of detailed quantitative data to obtain better flow descriptors for foam flow in porous geometry. In this work, through an accurate image processing and data analysis procedure that we describe in detail, we gain access to a variety of new data types for flow situations of this scale, displaying flow rates that are large (180 ft/day) but nonetheless realistic in proximity to the borewell. We present and evaluate the created flow maps in conjunction with the bubble size distributions. We develop new quantitative markers such as activated passage fractions and global flow distribution distributions. One novel aspect that we introduce is the inclusion of a numerical flow simulation performed on a 2D digitized version of the model we use. This provides a comparative Newtonian flow result, in order to asses tangibly the pore-scale specificities of foam flow versus a classical Newtonian fluid flow.

We witness some significant recurring themes, each representing a key observation in the study of foams in porous media. The structure of our discussion section follows a sequence of increasing complexity and fundamental explanation. We start by showing the interactions between the bubble size distributions and velocity distributions. With only these two parameters, we show that trapped foams are observed at a constant rate throughout the model and show independence to the surrounding flowing bubble size distribution. Secondly, we focus on the flowing foam population, and we show that within the continuum of speeds that contribute to flow, behavior differences are observed between bubble sizes. Here we establish the preferential paths for larger bubbles and demonstrate that the majority of flow in the medium is contained within these paths. Finally, we look in detail at the porous medium characteristics to attempt to explain some of the variance shown in the bubble flow maps, in both the trapped zones and preferential path zones. We perform a scan of structural properties 
demonstrating that local porous structure properties only weakly correlate with the flow properties and higher-complexity network properties are needed.

\section{Materials and Methods}

\subsection{Micromodel}

We use a glass micromodel of inner etched network dimensions of $10 \mathrm{~mm}$ wide by $17 \mathrm{~mm}$ long. The models are wet-etched with fabrication depth of $40 \mu \mathrm{m}$. Each outer longitudinal side of the inner sketching comprises a rectangular entrance zone followed by a flow diverting system of larger canals that serve the purpose of spreading the bubble flow across the model. The flow diverting channels are composed of 3 successive bifurcations of the initial entrance canal to produce 8 channels evenly distributed across the model entrance. Both sides of the model display the same flow spreading configuration inwards/outwards of the inner etching.

The interior model network is based on an image of a 2D slice of Bentheimer rock, obtained from X-Ray tomography. It is modified to include a larger degree of porosity and therefore connectivity, enabling a sufficient 2D percolation to correctly mimic a complex interconnected flow situation. We show a binarized version of the inner model used in Erreur ! Source du renvoi introuvable..

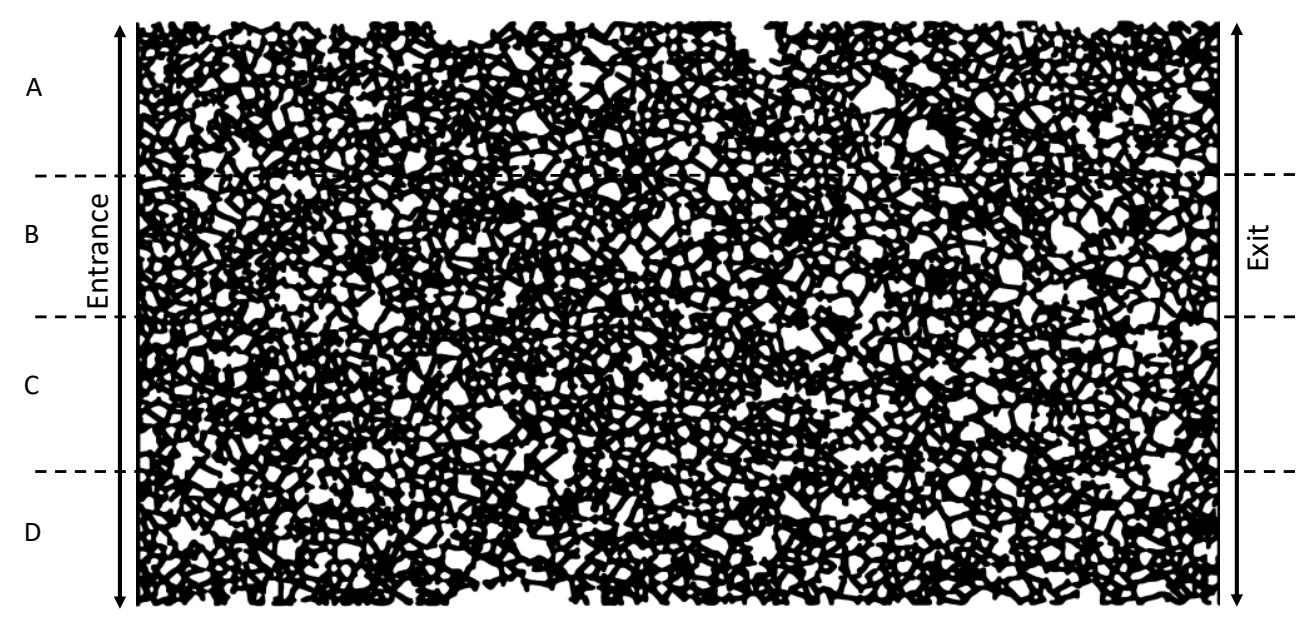

Figure 1: Binarized model showing the entrance and exit, as well as the zones used for the longitudinal box intensity analysis described in Appendix C.

The total amount of full-depth objects impeding flow (assimilated to grains) is 2602 . The average porosity of the interior connected network is $69.7 \%$. The permeability of the total model with the flow spreading system is 4.7 Darcy. As the model is made entirely from glass, it is water-wet. Using a simple adjustable watershed algorithm (Soille, Pierre; Vincent, Luc M. (1990)) on the binarized porous area of the model, local distance minima are identified, within a tolerance level parameter to the value 2 . The tolerance level value was set after comparison of different decompositions with varying tolerance values, settling for the decomposition that appeared most realistic. A sample of the chosen decomposition and associated statistics is shown in Figure 22. 

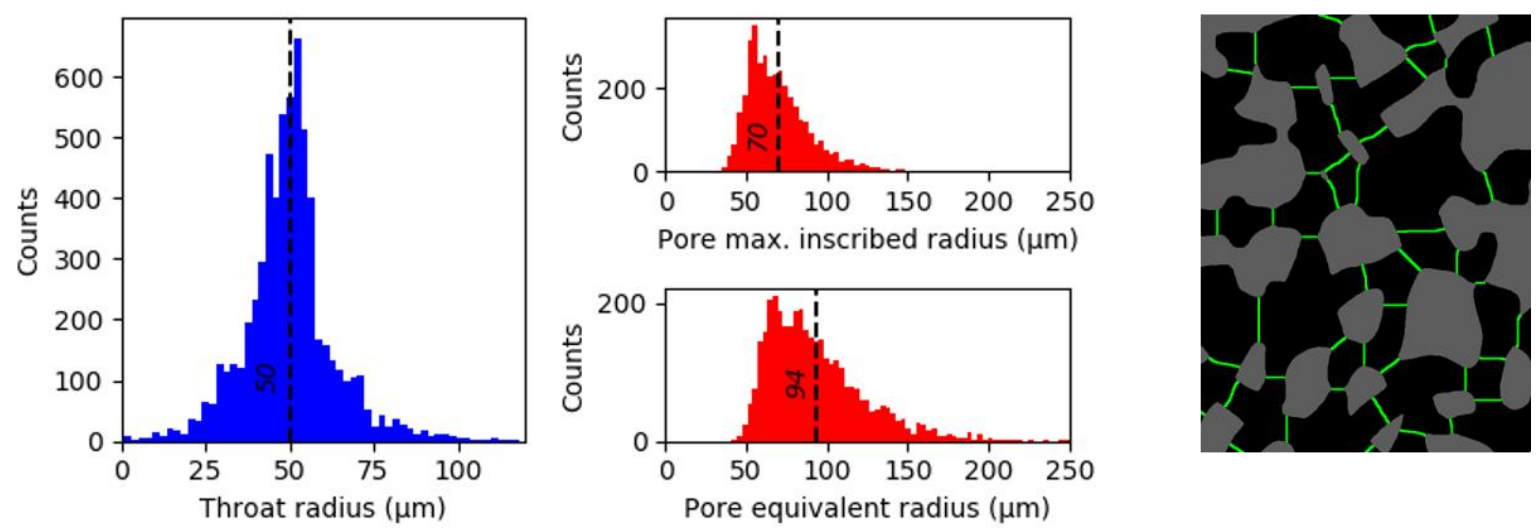

Figure 2: Micromodel network characteristics, with mean values shown as dashed lines. A sample of the network decomposition is shown on the right. Throats are shown as light green objects (enlarged for visibility). separating black pores.

From the watershedded model, we additionally use a specially designed network analysis code to extract neighborhood characteristics of the porous area. We obtain, for each pore, sizes of neighboring pores or throat sizes that lead to them.

\subsection{Fluids}

The gas used for the foam is purified $N_{2}$. The liquid component is composed of purified water, $30 \mathrm{~g} / \mathrm{L}$ of $\mathrm{NaCl}$, and an AOS 16-18 anionic surfactant at concentration of ten times the CMC, supplemented by a betaine-based foam booster known to improve the foamability, foam stability and rheological properties of aqueous foams (Elka S. Basheva, 2000). This solution has a surface tension in contact with air of 28.83 $\pm 0.01 \mathrm{mN} / \mathrm{m}$ at $24^{\circ} \mathrm{C}$, measured with a Kruss $\mathrm{K} 100$ tensiometer using the Wilhelmy plate method. This foaming solution has been selected due to its excellent stability and foamability, but also for the depth of literature available for AOS foams (Khatib 1986, Chambers 1990, Ehinger 1992 Gauteplass 2015), and the proximity of the salinity to that of seawater.

\subsection{Microfluidic setup}

In this section we describe the microfluidic setup that we have developed and tested. This setup includes an injection and monitoring system, with a high-resolution camera for direct observation of foam flow in the micromodel. The overall experimental setup diagram is shown in Figure 3. 


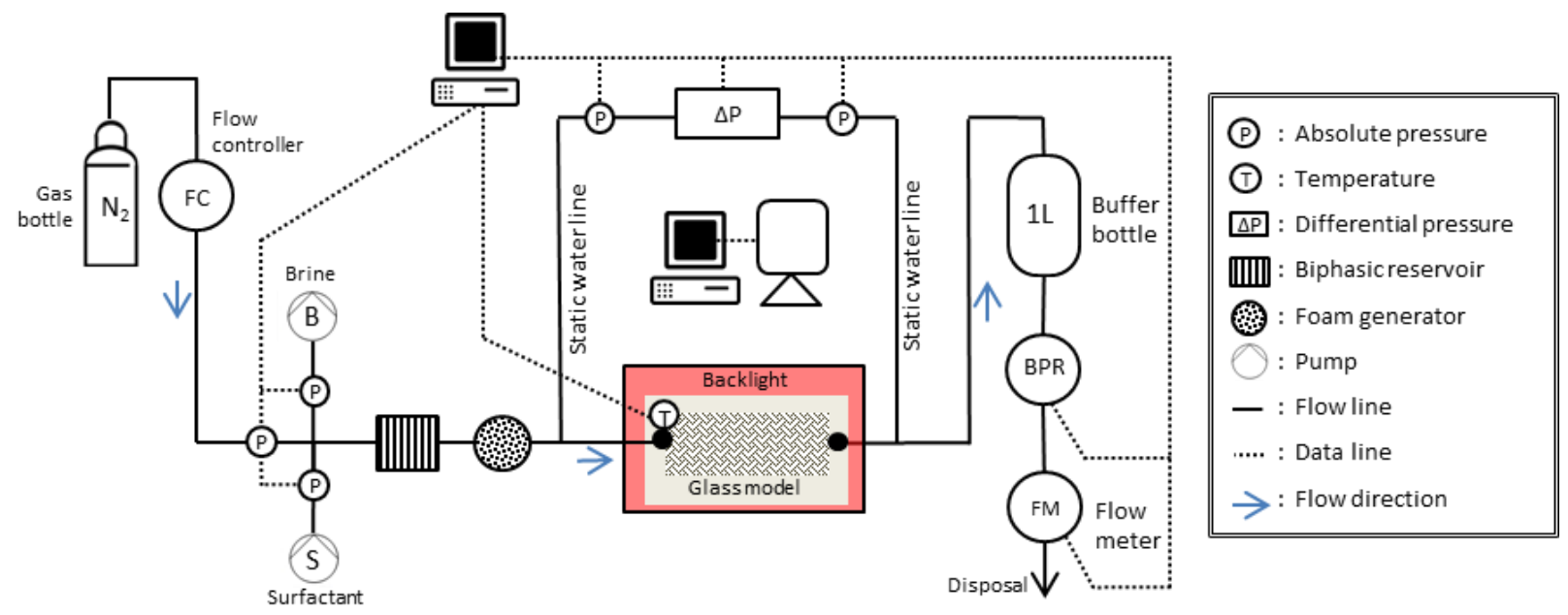

Figure 3: Experimental Setup

We use Vinduum liquid pumps, delivering a continuous flow and have a minimum volumetric delivery rate of $10^{-4} \mathrm{ml} / \mathrm{min}$. For gas flow, we use a Bronkhorst mass flow controller bounded by a maximum volume flow rate of $0.65 \mathrm{ml} / \mathrm{min}$ and a minimal stable flow rate found to be of $10^{-2} \mathrm{ml} / \mathrm{min}$.

The generation of foam occurs in a specific apparatus prior to the entrance of the micromodel. The foaming device is a sandpack of SiC grains $80 \mu \mathrm{m}$ in diameter. The sandpack is a cylinder $4 \mathrm{~cm}$ long and 1.5 $\mathrm{cm}$ in diameter, with a permeability measured to be $930 \mathrm{mD}$. The use of the sandpack is dual, firstly to provide a uniform foam quality before entering into the micromodel, as some supplementary segregation can occur in the tubing, and secondly to break down the gas-liquid biphasic flow into bubbles. Indeed, without creating preliminary coarse foam through the foam generator, we don't observe biphasic flow but rather a succession of distinct liquid and gas phases whose volume can reach similar a similar order of magnitude as the porous volume of the model. The foam injected into the model is thus both uniform and sufficiently coarse as to necessitate size re-adaptation through the passage in model. A backpressure regulator is used in the setup, fixed at 3 Bar, providing a means of minimizing compressibility effects from the created pressure gradient over the model. The high-frequency, high-resolution camera has a CCD acquisition with integrated RAM for a large image collection capacity, enabling capture of the whole model with part of the outer flow spreading systems, at a frequency of 605 frames per second and at a resolution of 2495 by 1496 pixels. The model is secured and levelled horizontally on two flat metallic rods, open to illumination from beneath. The model is backlit with the use of a high-intensity red light. In this configuration, the bubble interfaces are observable with a pixel size of $7.8 \mu \mathrm{m}$. Multiple thousand images can also be acquired in succession, all stabilised through the use of an air-suspended breadboard on which the setup is fixed, eliminating any potential laboratory vibration.

\section{Data acquisition and analysis tools}

\subsection{Experimental procedure}

The experimental procedure is designed to create a stable injection condition prior to acquiring steadystate images of flow that are representative of a unique gas fraction and injection rate. First, models are saturated with distilled water and an image of the clear model devoid of any foam is taken. A biphasic 
injection is initiated and surfactant solution and gas proceed to first through the foaming device before entering the model. As foam is created in the foaming apparatus, a significant pressure rise occurs at the entrance due to the viscosity of the created foam. In turn, foam propagation and further bubble size evolution across the micromodel create a pressure drop increase and is also tracked until a plateau is reached. The pressure at the foaming device inlet usually rises for a longer period of time than the model pressure drop and is also tracked until a plateau is reached. Only at this point is the system considered stable and images are acquired. For the experiment shown here, the injection gas fraction is set at $72 \%$, calculated at the exit of the model. At the time of acquisition, the steady pressure drop of $275 \pm 4 \mathrm{mBar}$, when compared to the backpressure of 3 Bars, ensures that the gas fraction is roughly the same throughout the model, varying from $70 \%$ at the entrance to $72 \%$ at the exit. The overall injection rate is $1.94 \times 10^{-2} \mathrm{~cm}^{3} / \mathrm{min}$, as measured at the model output at 3 Bars.

\subsection{Image acquisition strategy}

Two distinct image acquisition strategies are used. Each corresponds to a data type. In the case of the bubble size distributions, we average the individual distributions of images taken at a every 20 seconds. This low frequency was chosen such that multiple pore volumes of fluid could cross the model each time, keeping in mind the need for statistical representativity.

The images serving for dynamic bubble tracking and bubble creation observation were taken at higher frequency. Images sets are composed of 200 of these. Multiple image sets are captured, as one short burst of images may be insufficient to capture the sometimes fluctuating flow patterns. The number 200 was chosen firstly as the tracking process becomes somewhat computationally demanding after a larger number frames are used, and secondly as rather than a long single capture, many brief captures were preferred to account for the variability in flow patterns. Here we use 8 different sets acquired with a minute interval in between. A frequency was fine-tuned through trial and error to ensure we can track suitably all the bubbles (no blurring or excessive displacement between frames), while at the same time capturing the maximal amount of flow within 200 frames. The frequency was chosen as 605 frames per second.

\subsection{Image processing and exploitation}

\subsubsection{Image processing}

Our software analysis tools require binary images of filled bubbles. The grayscale images have to be preprocessed before binarization. Readers are encouraged to refer to appendix A for details regarding the image cleaning and binarization process.

\subsubsection{Measured bubble velocity map}

From the high-frequency image series, the bubble tracking procedure was performed and a large data set of individual tracking velocity points was produced. The tracking plugin gives both a value of bubble displacement between two frames and a local bubble area value. From the combined series of tracked bubble values we create a $2 \mathrm{D}$ flow map that gives a quantitative image of the flow over combined data series. The 2D flow maps are essentially 2D histograms of bubble velocities. The 2D bins for the image are 2-by-2 pixel boxes of the initial image. When a tracked object passes inside the box its velocity is added 
to the sum for that box. In this way, we observe a total velocity in a region in a given time, corresponding to a Eulerian description of flow. Each image is then smoothed using a Gaussian filter (implemented by a Gaussian kernel of the produced 2D array with a kernel with standard deviation of 3) as the particle-like nature of the bubble tracks can often create small contrasting patches inside a pore.

\subsection{Numerical flow comparison}

Throughout the study, a detailed flow comparison is consistently made with a 2D simulated flow of a Newtonian fluid in a 2D mask of the network. This comparison can enable the comparison of behavior between monophasic Newtonian fluids and foam, both on the pore scale and globally within in a complex network. The calculation was performed on the etched network only. The numerical method was a Lattice Boltzmann D2Q9 routine. The calculation was performed on scaled up (and interpolated) larger version of the model, for better spatial resolution, with a dimension of 8816 by 5109 pixels. The calculation was performed in about 143 hours at an average of 55 Million lattice updates per second, performing 570000 time steps until a steady velocity map was achieved and a quasi-constant pressure drop in between each timestep. The resulting velocity field output represents the Stokes flow of an incompressible Newtonian fluid. As the Stokes flow is purely viscous, the flow magnitude is isotropic. We give the velocity map of the simulation in Figure 6b.

3.5. Flow map quantitative analysis tools

In our study of the velocity maps, we make use of some other mesoscopic quantitative tools that support more firmly observations we can make from inspecting the maps. We define transversal passage activation and longitudinal box flow in detail Appendix B.

\section{Results}

\section{Bubble creation/destruction mechanisms}

The injected foams adapt rapidly to the porous network. Bubble creation is observed via the breaking up of large bubbles at bifurcations of the porous network. The division mechanism is described as follows. At a junction between one upstream path and two downstream paths an upstream large bubble enters partially one of the downstream paths, but is it broken up by a following bubble that breaks off the trailing part into a different path. This can also be assimilated to neighbor-induced creation mechanisms due to the high injection rate as previously observed (Liontas 2013). We observe that if the part of the larger bubble that is committed to the trailing path is not sufficient large, it will not get broken off, leading to the bubble deforming around the bifurcation and keeping its integrity. An example of both a successful division event and a failed division event is observed in Figure 4. We understand that capillary snap-off is unlikely to be observed in a model of this given geometry, due to the required large aspect ratio in a 2dimensional medium that we observe rarely in our network (Rossen, William R. (2008)). For this reason the bubble fractioning dynamics has not been studied extensively, as is the case in other studies (Géraud 2017). 

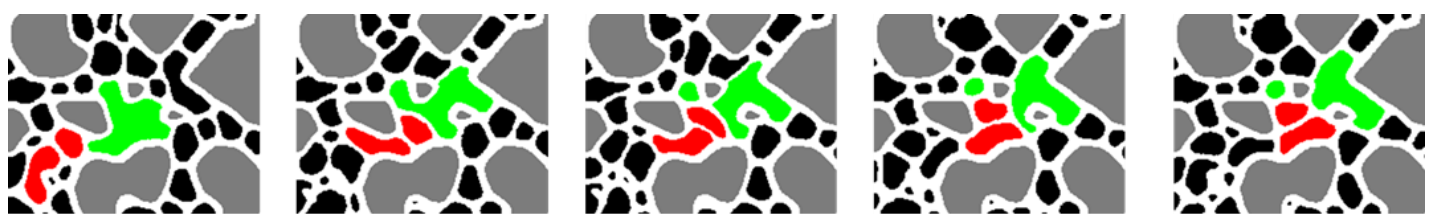

time

Figure 4: Observation of a successful bubble division (green bubble, left pane to center pane), followed by an unsuccessful one (lower red bubble, center pane to right pane). The red bubbles are responsible for pushing the green bubble against a bifurcation. The time between each pane is $3.3 \mathrm{~ms}$.

Bubble destruction or destabilization was not observed at these experimental conditions. This could reflect the important liquid fraction of the flow, constantly providing surfactant for film stabilization, or otherwise could be due to the low average aspect ratio of the model. Indeed, a film exiting a throat and expanding into a larger pore will only thin and destabilize if the downstream pore is large enough.

\section{Bubble size distributions}

From the low-frequency image series, the images are cut in five sections transversally, from the entrance to the exit of the model corresponding to the first fifth of the model after the entrance, the second fifth etc. up to the final exit box. The goal is to probe the evolution of bubble size throughout the model. The binarized bubble areas are measured. Each area is then associated to an equivalent radius, through circular approximation. The measured bubble radii for 20 different images is then represented in a weighted histogram of 70 equally spaced bins from 0 to a value at which bubble detection becomes negligible. The value in each histogram bin is then multiplied by the center value of the bin, producing a weighted histogram that shows the area occupied by each bubble radius, rather than a simple number count. Through this approach, the histogram then can be associated with a probability density of choosing a given bubble size when choosing a location at random in the porous space. We display the produced bubble size distributions in Figure 5. 


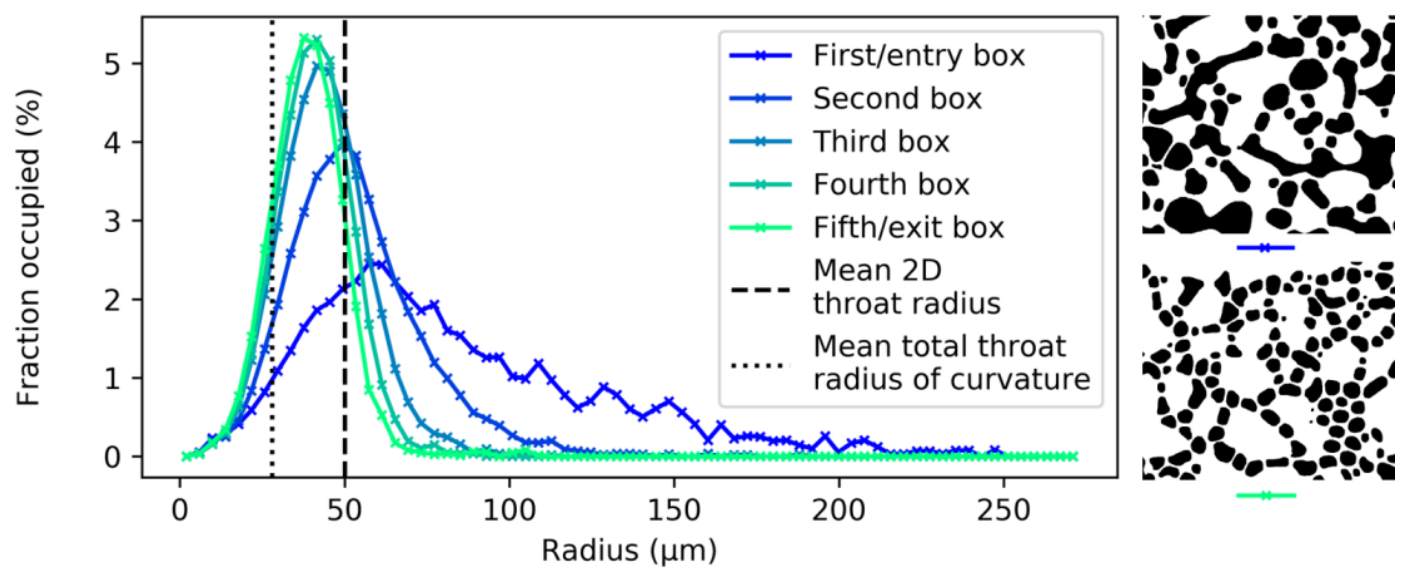

Figure 5: Bubble size distributions for different transversal sections of the model. A small example of the processed bubble images is included for the sections closest to the entrance and exit.

The bubbles contained in each box are shown in a different color on Erreur ! Source du renvoi introuvable. 5 ranging from blue (entrance of the model) to green (exit of the model). We display the average $2 \mathrm{D}$ throat radius (as shown in Figure 2 ) as well the average total throat radius of curvature, given by $2\left(\frac{1}{r_{t}}+\frac{1}{r_{d}}\right)^{-1}$, where $r_{t}$ is the throat radius and $r_{d}$ is the radius associated to the model depth, or $20 \mu \mathrm{m}$. We observe an evolution in bubble size from large bubbles at the entrance to a smaller, spiked distribution at the end section. The evolution occurs mainly over the first two sections after which the distribution seems to evolve only marginally. Furthermore, the end distribution appears to be symmetrical, with no presence of a right hand side tail that would account for larger bubbles. We expect that a longer model would further increase the monodispersity of the bubbles. Intuitively, the average throat size (as shown in Figure 5Erreur ! Source du renvoi introuvable.), or the average total throat radius of curvature, could be seen as the limiting dimensions in the system, expecting the peak of the bubble distributions to be situated on either of them. However, the peak of the final bubble distribution is situated in between both averages. Due to the neighbor-induced nature of the bubble creation mechanism, the injection rate obviously plays a part in the final bubble size distribution. A higher injection rate will allow less time for a trailing part of a bubble to deform around a bifurcation before a trailing bubble will break it up; failed divisions, such as observed in Figure 4, would become more rare. The bubble sizes lower than the average throat value are therefore linked to the high injection rate of the experiment.

\section{Comparison of velocity maps}

We show in Figure 6a the overall measured velocity map for the experiment, for all the bubble tracks over the 8 datasets of 200 images, as described in Appendix B. The map shows both the velocity field, given in detail by the color-bar of Figure 8 , and solid elements of the model in dark grey. Looking at the map 
globally, we note a large number of similarities with the Newtonian flow simulation displayed in Erreur ! Source du renvoi introuvable. We also display the velocity map for the $20 \%$ smallest (Figure $6 \mathrm{c}$ ) and $20 \%$ largest (Figure 6d) bubbles, explored in greater detail in the discussion section. For the overall velocity map (Figure 6a) we observe a large number of passages in use. On top of this, the flow seems to be well distributed throughout each section of the model, similarly to the Newtonian simulation. In Figure 7a and $7 b$, we observe that this is also quantitatively true.

a)

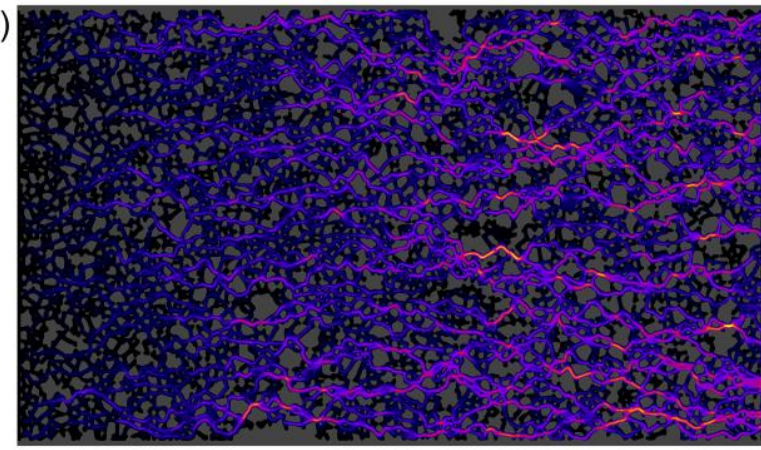

c)

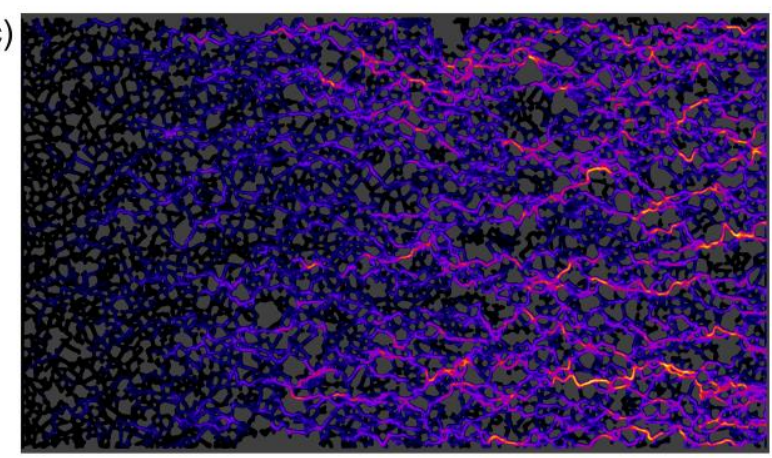

b)

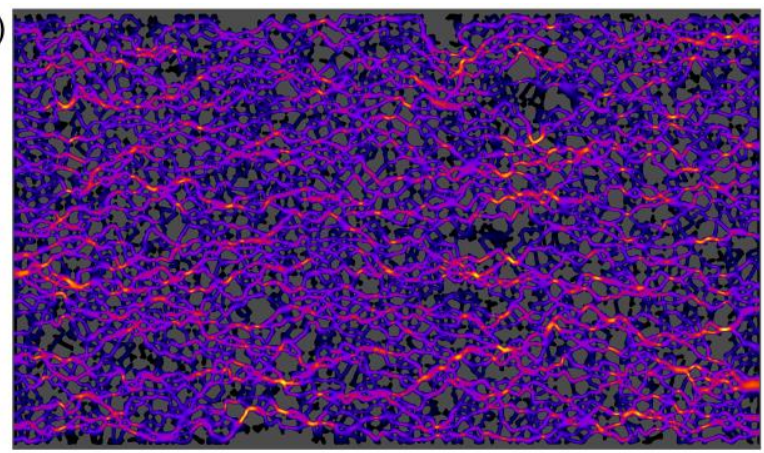

d)

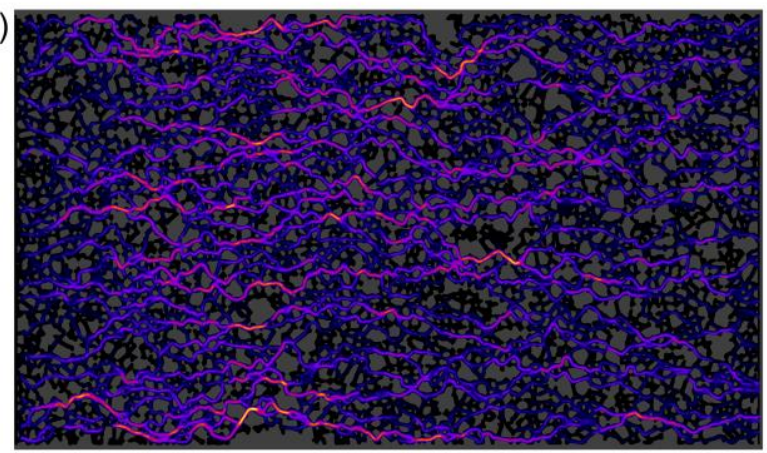

Figure 6: Different velocity maps using the same model. Injections are from left to right. a) Foam flow, all bubble sizes. b) Numerical Newtonian flow. c) Foam flow, smallest 20\% of bubbles. d) Foam flow, largest $20 \%$ of bubbles.

This global similarity is confirmed by the breakdown of the flowmaps into the velocity components parallel and perpendicular to pressure gradient, as these datatypes are available in both the bubble velocity tracking and the Newtonian numerical case. We produce velocity maps for each component in each case (not shown here), and sum the absolute value of the image intensity of the entire image to compare values. The flow breakdown in $\mathrm{x}$ (parallel to pressure gradient) and $\mathrm{y}$ (perpendicular to pressure gradient) directions are comparable in both cases, as shown in $7 \mathrm{c}$. 
a)

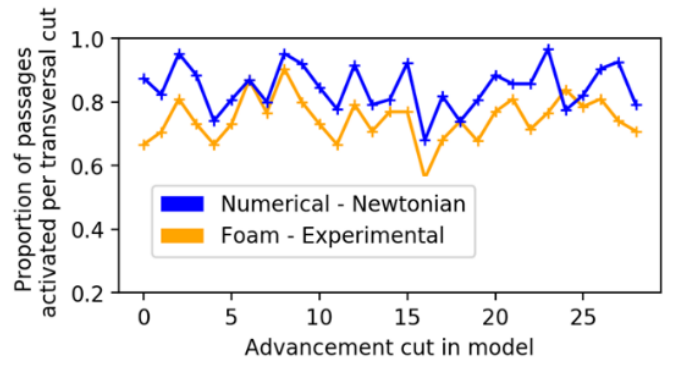

b)

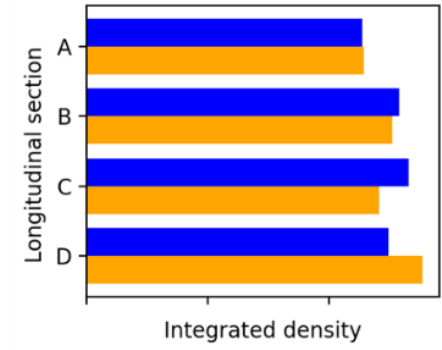

c)

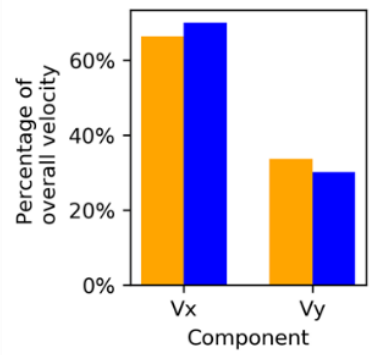

Figure 7: Quantitative comparison of numerical Newtonian flow and measured foam flow.

At the pore-scale, further common characteristics are observed. Preferential paths - appearing in bright colors - emerge in both cases. These paths are aligned in the direction of the pressure gradient which is directed here from left to right. The Erreur! Source du renvoi introuvable.8Erreur! Source du renvoi introuvable. illustrates the two different flow situations on a smaller scale.
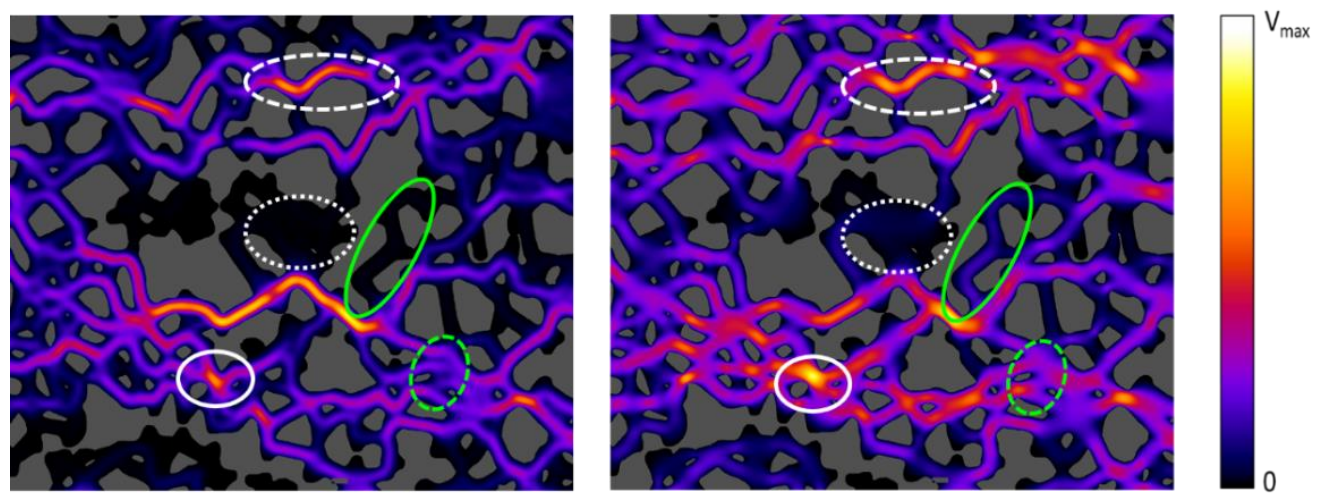

Figure 8: Detailed comparison of experimental (left) and numerical (right) flow, showcasing a series of similarities and notable differences. Flow is left to right.

The presence of zones devoid or low in flow, visible by the absence of color (surrounded by a thinly dashed oval), exist in both measured foam flow and simulated Newtonian Stokes flow. In the foam experiments these zones are filled with trapped bubbles. These zones are often found beside path junctions comprising large amounts of flow (surrounded by a solid oval). Also, longer paths with conserved flow are visible that span multiple network bifurcations (surrounded by a thickly dashed oval). 
However, the microscopic differences in the comparison we give here are not negligible. Overall, a larger degree of flow heterogeneity appears in the experimental image, shown by the higher contrasting colors. In detail for example, while the flow is non-existent in the dead zones in the foam experiment, the Newtonian flow situation shows a residual amount of flow in the central marked zone. We can observe that some passages that turn away from the flow direction (full green ovals) are completely inactive in the foam experiment, while showing some flow in the Newtonian case. Also, the foam flow in larger pores is sometimes represented by more than one trail (dashed green ovals), as opposed to the diffused continuous signal in the Newtonian experiment. This is due to the fact that multiple bubbles flow simultaneously into the larger pores, originating from smaller upstream passages.

\section{Discussion}

\section{Bubble velocity and size relationship}

It has been previously established (Géraud, 2015) that a strong positive correlation exists between bubble size and bubble velocity for foam flow in a $2 D$ analog porous network, specifically for high-gas fraction injections. Our data follows this trend. Figure 9 displays density plots of the bubble speeds in terms of the bubble sizes, for different parts of the model, that is cut into five equal sections (full model, entrance section, exit section).
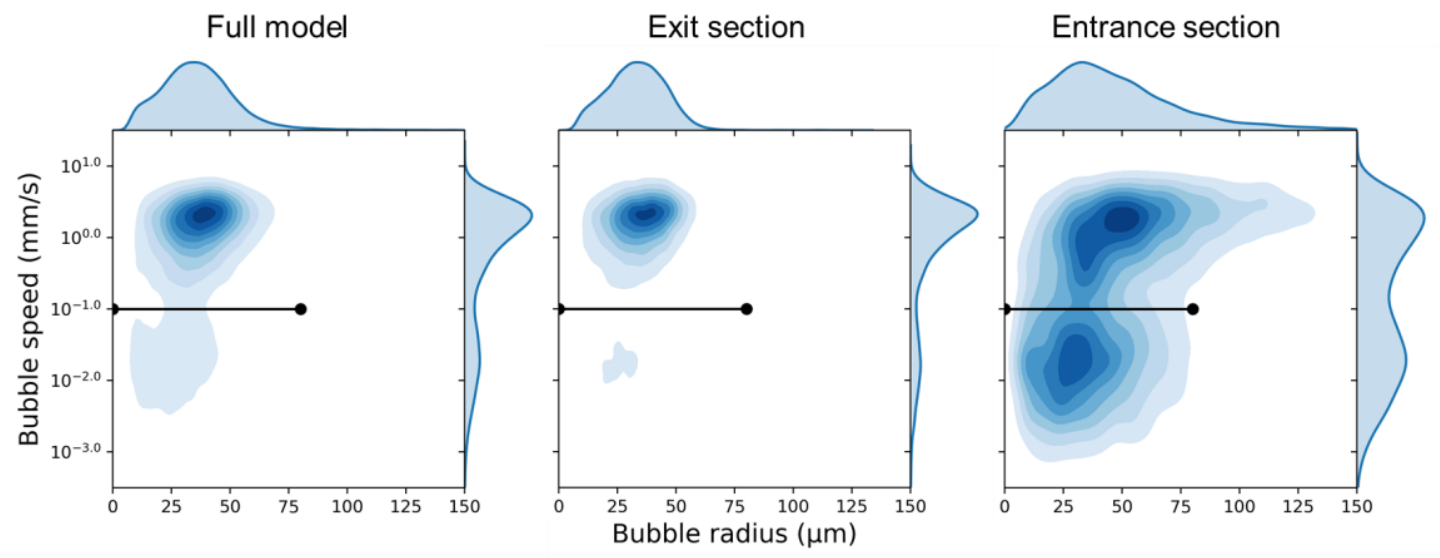

Figure 9: Density plots of bubble speeds and bubble radii in different sections of the model. We observe two distinct populations corresponding to trapped bubbles (lower population) and flowing bubbles

(higher population). The density map on the left is created with 82623 individual points, each representing a bubble track (50 frames minimum), the central plot is made with 8664 and the plot to the right with 20406 points, demonstrating the unequal number of bubbles in each section. The speed is given by the Euclidean distance of the bubble from the final to initial frame, divided by the bubble track length.

The size is the average size over the bubble track.

In the full model density plot (Erreur ! Source du renvoi introuvable., left), the main cluster of points shows a weakly positive relationship between bubble size and radius. However, a second, distinct cluster of small, low-speed bubbles exists in the lower left corner of the plot. These points are trapped bubbles. 
When examining similar plots for each transversal section of the model we observe that the trapped fraction of bubbles are represented more clearly in the entrance of the model (Erreur ! Source du renvoi introuvable., right), whereas flowing bubbles appear more clearly at the end. To differentiate between both populations we divide the plot using a visually chosen line in showed in black. As the density plots display the relative distribution of points and not absolute numbers, to complement these observations, we display in Figure 10 the number of points contained in each side of the black line, for each transversal box of the model, following the progression of the flow.
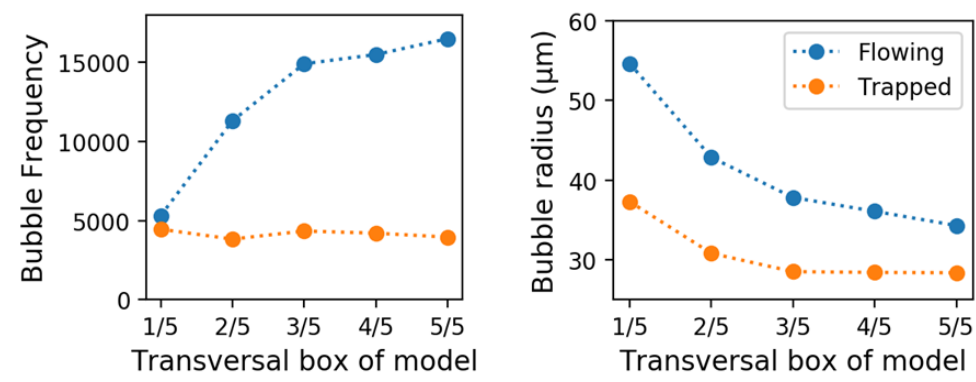

Figure 10: Frequency of bubbles and average radius in each bubble population for each transversal box of the model. Note that here the average bubble size is calculated simply over all bubbles. The discrepancy between the peak of Figure 5 (which displays a weighted histogram, rather than a number frequency histogram) and the average value show here is well understood.

Erreur ! Source du renvoi introuvable. shows that while the number of flowing bubbles tends to increase as large bubbles divide up as they pass through the model (18000 at the end vs. 5000 at the beginning), the number of trapped bubbles remains relatively constant in each part of the model. Also we see that the average bubble size in the flowing foam evolves all the way through the model whereas the trapped foam size is relatively constant after the second section. These opposing behaviors reaffirm the distinctiveness of the two populations. The implications of these observations are significant. The number of trapped bubbles is constant in a homogeneous medium, independently of the surrounding flowing bubble distribution. Furthermore, as the bubble size is relatively constant, we can affirm that the trapped fraction (porous space filled with stationary foam) is also constant despite a continually evolving bubble size distribution elsewhere. While the observation of inference of a trapped foam fraction is shown elsewhere (Jones 2017, Nguyen 2009, Nguyen 2002), local evaluation of the trapped fraction and its independence to the surrounding flowing foam size distribution is yet to be observed. We believe the trapped foams seen here will not contribute greatly to the macroscopic steady-state flow properties (small contribution to the apparent viscosity) as they are situated in hard-to-access zones. However, as they can enclose oil in pseudo-emulsion films between the oil-gas interfaces (Bergeron 1992), and deplete surfactant, the small trapped foams should be considered for simulation of foam flow.

We further propose a causal link between the trapped bubble sizes and velocities. We observe multiple mechanisms that contribute to the population of trapped zones by small bubbles. First, we note that the smaller bubbles are more likely to move off the preferential tracks into low-flow areas. The image panels in Figure 11 demonstrate our argument, in which we show this mechanism repeating itself twice in the same location in a time frame of $330 \mathrm{~ms}$, or a single dataset of 200 images. In these images, we overlay flow lines on images of bubbles (shown in color), whilst the solid obstacles are shown in gray. Flow line thickness shows flow intensity. Flow lines were calculated using quantitative data from the combined datasets. We see that two smaller bubbles, shown in red, move into a low-flow zone at the bottom of the 
image, characterized by an absence of measured flow lines. We observe that larger bubbles, shown in green, feel contained by other bubbles dragging and pushing them to keep to the main flow paths. Indeed, as they cover a larger area, they are more likely to intersect a high flow path, despite part of their interface exploring a low-flow zone. Inversely, once smaller bubbles explore low-flow regions, they can be rapidly locked out. Combined with the fact that their displacement into low-flow zones requires less displacement of static fluids, their chances of successfully entering and staying in the low-flow areas are increased.
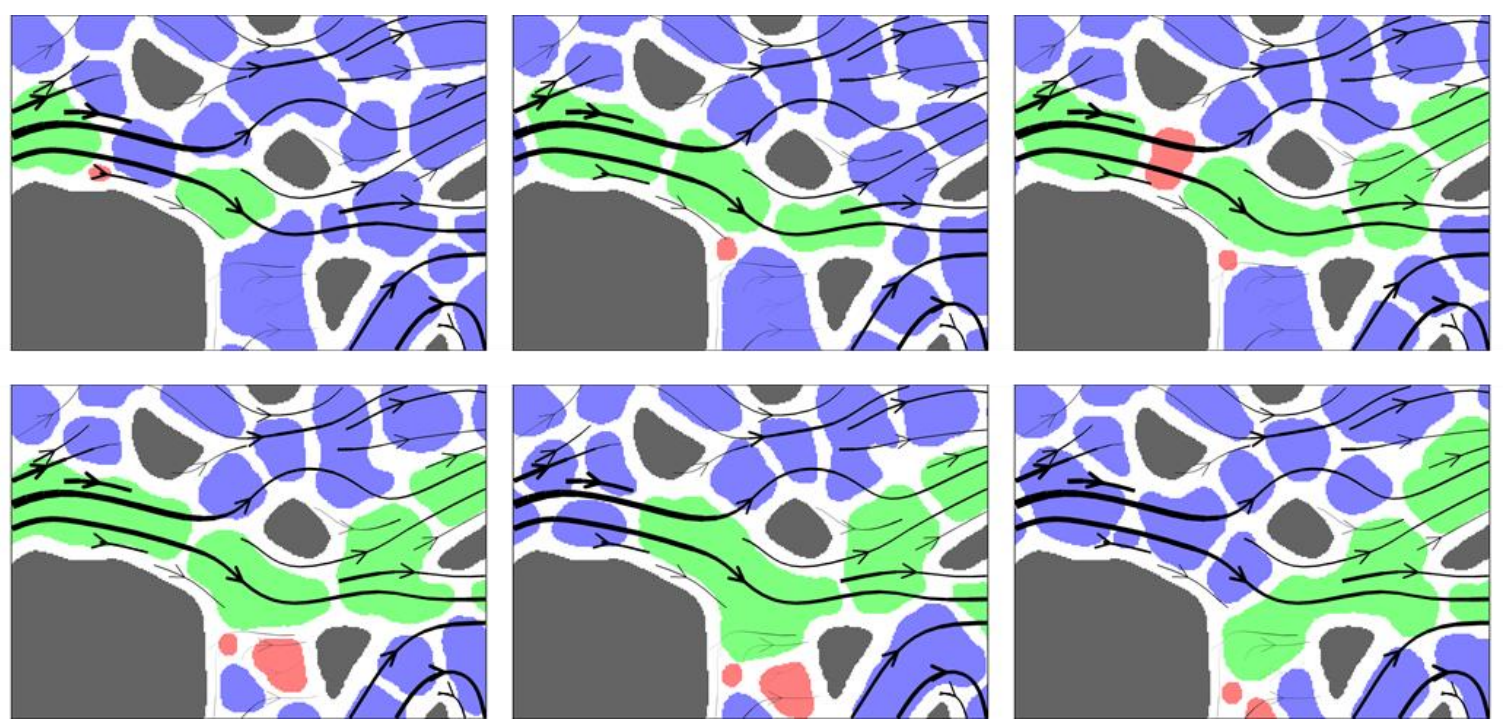

Figure 11: Small bubble trapping mechanism. Flow lines are calculated from the combined datasets. Smaller bubbles trapping are shown in red, whilst larger bubbles keeping to the main path are shown in green. Solid obstacles are shown in gray. The time between each panel is $66 \mathrm{~ms}$.

Secondly, we also observe a large amount of trapped bubbles in pore corners and dead end pores. Examples of both are given in Figure 12, in which we show image averages for two locations for a dataset of 200 images. The black isolated bubbles show that they are not displaced during the entirety of the dataset, whilst gray values in the flowing areas show a succession of distinct bubbles.
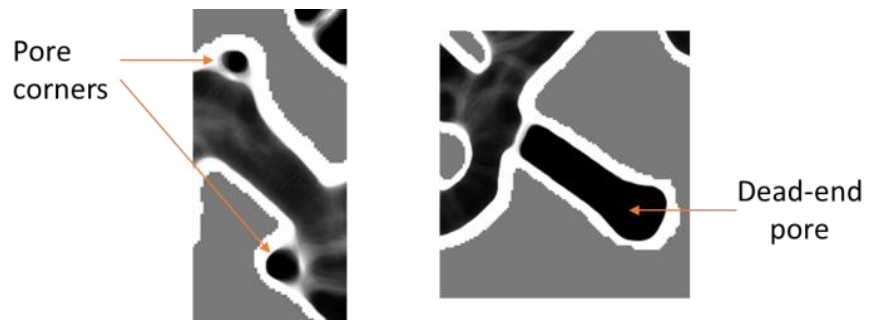

Figure 12: Trapped bubbles in pore corners and dead-end pores. An image set of 200 images is averaged to show flowing areas with varying shades of gray. The solid obstacles are shown in gray.

Indeed, while low-flow zones, as described above, are structurally connected to the rest of the network in at least two ways, pore corners and dead end pores have only one outside connection. As bubbles in these areas tend to trap and rarely displace, observation of their specific trapping mechanism is not evident as datasets are acquired in a steady state pressure gradient when flow distribution is well established. However, we argue that for the case of dead-end pores, the trapping mechanism is the 
creation mechanism described previously, where part of a larger bubble is ripped off in situ and occupies the dead end. Indeed, these bubbles fill the dead-end pores entirely and seem to have been tailored for the pores precisely. A few large, trapped bubbles have hence been observed in some larger dead-end pores. Oppositely, trapped corner pore bubbles tend to be a lot smaller, and can be created elsewhere in the network that have found a stable position where they are unaffected by flow. In fact, the possibility of creating a pore corner bubble in situ seems unlikely as the network does not offer a sufficiently deep bifurcation for larger bubbles to invade and get broken apart by trailing bubbles. The number of smaller, corner pore bubbles greatly outweighs the larger dead-end pore bubbles.

Obviously, it can be difficult to differentiate between qualifying some areas as a pore corner or a deadend pore, but the distinction is worthy as we believe these areas become populated with trapped bubbles in different ways.

\section{Preferential path flow for larger bubbles}

Here we take a closer look at Figure 6 in which are displayed velocity maps for different bubble size populations. The most striking observation is the differences in velocity intensity gradients appearing over the model in the flow direction. In reality, this simply translates the different amounts of measurement points contributing to the 2D histogram, and is another manifestation of the bubble size adaptation. This explains the stronger strength of the intensity gradient for the smallest $20 \%$ image (Figure $6 \mathrm{c}$ ), as this bubble size is inexistent at the entrance of the model, but prevalent near the exit. Inversely, the larger bubbles are seen most at the entrance. The second observation is the difference in flow patterns between the largest (Figure 6d) and smallest (Figure 6c), bubble sizes. Broadly speaking, the larger bubbles take paths more parallel to the pressure gradient and are present in less paths overall. Also, for larger bubbles, the notion of large-scale path seems to be more significant, in other words, flow intensity is conserved over a single path that can span many pores and throats, and not diffused out into every possible downstream avenue. The smaller bubble flow (Figure 6c) is also present in preferential paths as small bubbles are carried along with larger bubbles, but also is visible in many zones unused by the largest bubbles. These paths are often perpendicular to the pressure gradient, and are accessed by smaller 
bubbles through the mechanism displayed in Figure 11. The contribution of each component (longitudinal, $\mathrm{Vx}$ and transversal, $\mathrm{Vy}$ ) of the velocity maps for each bubble size category is shown in Figure 13.

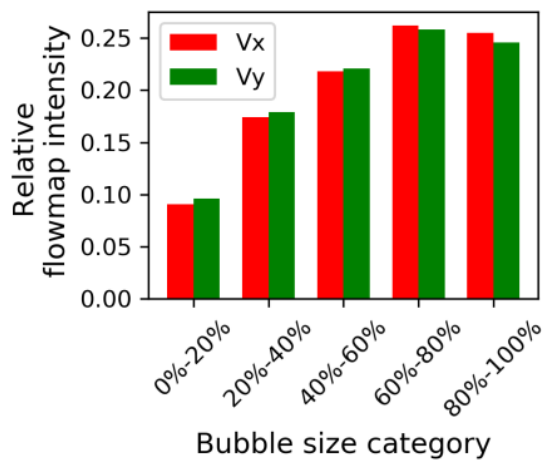

Figure 13: Contribution of longitudinal (Vx) and transversal (Vy) velocity components for each bubble size category for the experimental foam data. Note that the overall magnitude of the sum of each component has been normalized for clarity, overall comparison of magnitude of each component is shown in $7 c$.

The flowmap intensities shown here are given by the sum of pixel values for each map, as individual maps were obtained as described for the comparison of Figure 7. The values are then normalized for each component. We remark that an increased amount of flow is carried by the larger bubbles $(60-80 \%$ and 80-100\%), despite the well-understood overrepresentation of smaller bubbles in the maps due to their increased number of observations, owing to their smaller size.

Finally, we note that each velocity component makes a similar relative contribution for each bubble size. This seemingly paradoxical result demonstrates that large-scale preferential paths are not chosen on a basis of immediate local preference for pores in parallel to flow (which would imply a higher relative longitudinal velocity contribution) but instead a more global idea of path shortness or straightness in which flow is not restrained to the longitudinal component. Specifically we predict that the preferential flow paths are created via a tradeoff of ease-of-flow (through large throats or pores) and shortness of the overall path length. We further explore the idea of global paths in the following section of our discussion.

\section{Local structural relationships}

Finally, the relationship between measured bubble velocity and structural elements of the porous medium was explored. Géraud (2016) find a positive correlation between the average velocity and the pore size in the medium. Regarding preferential paths, they affirm "that most preferential paths occur where a series of large pores are connected together". Through this statement they include a notion of both network properties and local pore size. Subsequently, they offer a link between structural elements and trapped foam areas via "bubbles trapped in regions of low flow velocity in-between closely set grains". However, it is unclear whether "closely set grains" relates to a small pore size or small throat entrance.

To explore this observation for our data we must first apply some supplementary steps to our experimental image, as it shows a significant intensity gradient due to bubble size evolution, which serves 
as an unfair basis for local flow intensity comparison. We refer the reader to Appendix $\mathrm{C}$ for further detail regarding overall intensity uniformization procedure.

For our structural decomposition in terms of pores and throats we use the same watershedded image as described in Figure 3. To achieve a comparable intensity value for each structural element of the medium (pore or throat), we calculate an average intensity value per element.
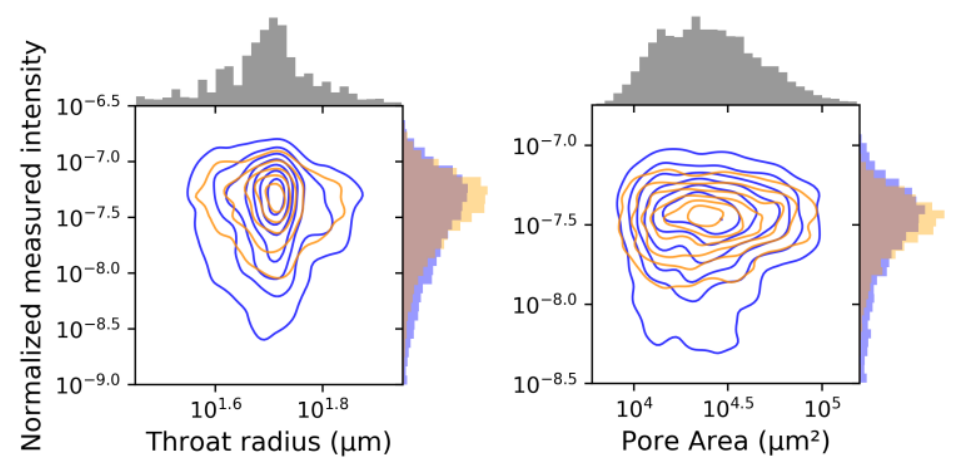

Figure 44: Normalized per pixel intensities for throats and pores in the numerical Newtonian flow (orange) and the experimental foam flow (blue) shown as density contours.

We show in Figure 14 contour maps comparing the observed average intensities in each pore and throat for both flow maps (numerical Newtonian and experimental foam), accompanied by the histograms on the opposing spline of the plot of both the structural parameters and the average measured intensities. The pore and throat contour maps were created with around 3500 and 6000 points respectively, as some outliers are excluded for plotting purposes. For each color in each plot, 7 distinct contour lines are plotted. In both throat and pore comparisons, we see a similar behavior for both flow situations; the distribution of average intensity values seems to be fairly independent of the pore or throat size, showing a symmetrical distribution around the average pore and throat size. However, the foam contours span a larger range of measured intensities for both throats and pores. This indicates a more heterogeneous foam flow, confirming our previous observations; the lower contours of the foam experiment (reaching much smaller values of intensity) can be in part explained by the presence of dead zones in the foam flow (cf. Figure 8, areas that display little or no flow).

Pore or throat size, does not, on average, correlate with flow properties. Although some weak trends may appear, the data dispersion is too high to show a solid relationship. Further investigation of more complex local structural parameters, including parameters derived from the descriptions of Géraud et al. (2016), regarding the preferential paths and trapped zones, can be found in Appendix $D$. The conclusions are identical to those above, notably a lack of overarching correlation between local structural properties related to pore and throat sizes and flow properties.

Our explanation for the lack of correlation between local structural properties and foam flow intensity is the higher network complexity of our model, i.e. the larger number of elements that make up its length. We show that in realistic networks with a high number of structural elements and possible paths, local relationships are inadequate for flow prediction. Indeed, the possibility of flow in a given element is a function of the upstream and downstream elements that surround it. A large pore with a dead-end path downstream will not contain any flow, just as no flow will access a hard to reach pore from upstream. The higher the network length, the higher the probability that flow may be impeded by a structural element 
some distance away. In this sense, only through a model with a length and complexity as the one shown here does this distinctive path-dependent behavior become evident. In this regard, we explore the dependence on the pore coordination number, which is given by the number of first neighbors each pore possesses. We display the results in the form of a boxplot in Figure 15.

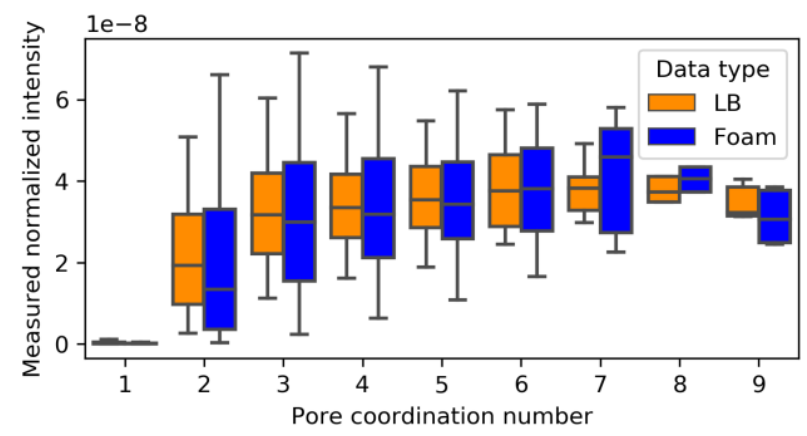

Figure 15: Measured per pixel intensity for pores with varying pore coordination numbers, for both numerical Lattice Boltzmann (LB) and foam experiments.

For each box of the plot, the median is shown as a black bar in the middle of the box, separating the data in half; the edges of the box represent the upper and lower quartiles of the data, and the end of the whiskers show the extent of the other data points, excluding the upper and lower outliers, or $5 \%$ of the data on each extreme. Again, the experimental data shows a larger distribution of intensity values for each pore coordination value. Both cases show zero flow for pores with a coordination value of 1 , or deadend pores. However, it's worth noting that the median for the foam experiment rises faster than the Newtonian counterpart, owing to a larger dependence on pore coordination in the case of foam. This implies that for a realistic microscopic prediction of foam flow in a complex porous medium, pore coordination must be considered to a larger degree than in a Newtonian flow situation, and significantly, flow prediction in a complex medium must necessarily integrate abstract network elements such as coordination rather than focusing on local properties of size.

\section{Conclusion and perspectives}

Through this study we integrate new quantitative tools to assess high-velocity, high-liquid fraction foam flow behavior in relation to a Newtonian viscous flow comparison. We gain access and interpret highresolution unseen data at this scale for foam studies. Overall we find a large degree of similarities in our global and local comparison of data in both foam and Newtonian situations, including comparable overall passage activation, flow distribution and component-specific velocity distributions. Despite this similarity, the foam flow displays a more heterogeneous array of flow situations. Specifically, elements that were observed and analyzed only in the foam case include trapped foams and preferential paths. Notably, we deepen the link between trapped foams and bubble sizes, showing a distinct population of immobile small bubbles exists throughout the model, displaying independence to the surrounding bubble size distribution and to the location in the model. We also observe the existence of high velocity preferential paths, serving as the unique transporting zones for large bubbles, whereas smaller bubbles can either be transported in preferential paths along with the larger bubbles, or in either hard-to-access paths or paths perpendicular to the pressure gradient. Finally, we pursue a naïve attempt of flow prediction in terms of local 
parameters. From this analysis we conclude that local structure only weakly predicts flow properties and that to fully grasp the notion of preferential paths and trapped areas from a structural point of view, it is essential to integrate notions related to network properties into the prediction process. Attempts at modelling foam flow in porous media microscopically must integrate the notion of long-range preferential paths. Furthermore, we can imagine that a characterization of the preferential paths is a prerequisite for linking porous microstructure to macroscopic properties in foam flow such as apparent viscosity. 


\section{Appendix A: Image processing workflow}

The following processing procedure is designed to maximize the contrast between the gas bubbles and the liquid-gas interfaces that separates them before binarization. All scripts were written in ImageJ macro language.

The first step is the subtraction of the image of the clear model from the foam image. Subtraction of the initial saturated model therefore creates more uniformity for the gas-liquid interfaces we are interested in. Removal of the solid-liquid interface also ensures that liquid-filled spaces bordering the grains are eliminated. The only remaining contrasting elements are the gas-liquid interfaces. Subtraction of the liquid filled model also serves to erase any illumination heterogeneity that may be created by the backlight system. This step is illustrated in Figure A-1.

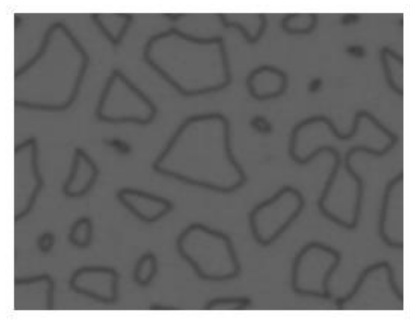

a)

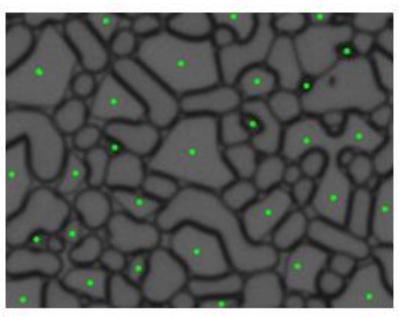

b)

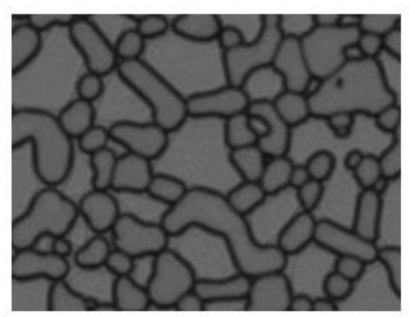

c)

Figure A-1: Background removal process. a) The initial brine-saturated model. b) The model with foam before the subtraction, grains marked with green dots. $c$ ) The result of the subtraction of a) from $b$ ), removing all solid interfaces and homogenizing gas-liquid interface gray value.

We then locally increase contrast through the use of the built-in imageJ function Enhance Local Contrast (CLAHE) (Zuiderveld, Karel (1994)).

After thresholding the constrasted image, the next step of the process is isolating bubbles from the grains. For the solid grain removal we make use of the liquid-filled image in A-1a on which we apply the default threshold. The resulting binary image of solid-liquid interfaces is then filled. We use the "Ultimate Points" process to return an image with the location of the Ultimate eroded points, representing the centers of particles that would be separated by a local-minima based segmentation. We then filter out the unwanted objects using the Morpholibj plugin (Legland, D.; Arganda-Carreras, I. \& Andrey, P. 2016) that allows morphological reconstruction of a binary image from a series of "seeds", given by the Ultimate points. The reconstructed grains and their contours are subsequently removed from the thresholded foam image to leave only the foam bubbles. Figure A-2 shows this process in detail. 


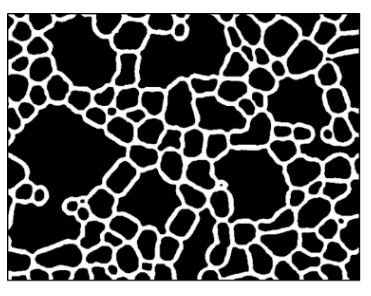

a)

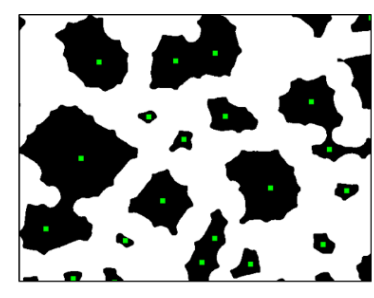

b)

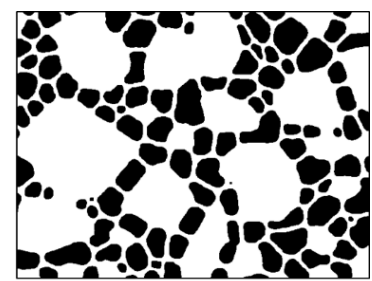

c)

Figure A-2: Image processing steps showing the removal of unwanted grain objects from an initial

binarized image. The initial image is shown left. Grain objects are identified using Ultimate points (shown as green points) from a binarized image of the model and are reconstructed in the original image using morphological reconstruction (center). The unwanted objects are subtracted from the initial image (right).

For the dynamic tracking, we use a plugin (Nussbaum-Krammer et al. 2015) to track the bubbles through the successive frames, obtaining precise paths and velocities amongst other observations. The bubble tracking method works in a simple manner, following each object frame-by-frame by evaluating the closest positioned and similarly sized bubble in a successive frame. When multiple candidate objects are equally viable in a successive frame, the algorithm puts a flag on the potential object and works on retrieving other surrounding bubbles first, in hopes of eliminating ambiguity when the flagged object is re-evaluated. Despite its simplicity, the algorithm works surprisingly well and is reasonably fast considering the large amount of densely packed objects in each frame (around 20 minutes for more than 10000 bubbles). The accuracy of the method owes to the high frequency acquisition. The small bubble displacement between each frame generates low ambiguity in bubble identification. However, the algorithm requires the input of a few parameters. These parameters serve as a means to narrow down potential candidates in the neighbor-evaluating process.

The first parameter is the maximum tolerated bubble area change. This value was fixed at $50 \%$, both to allow for bubble compression and bubble subdivision with flow. In the case of a bubble division, the smaller broken off bubble is considered as a new object with its own distinct track and the larger parent is retained in the original track. Multiple subdivisions of an initial bubble in the same dataset are rare. The second parameter is the minimum number of successive frames in which a bubble needs present. This parameter was fixed at 50 frames. The last tunable parameter in the tracking process is the maximal allowed displacement per bubble in between frames. This value was chosen from inspection of the frameby-frame bubble displacement histogram created by initially letting this parameter take an unphysically large value. When doing this, the algorithm returns a negligible number of bubble displacement measurements all the way up until the unphysical limiting value. Upon examination, displacements at this speed are erroneous and correspond to jumps between bubbles. The choice of the final parameter value is then chosen in accordance with the displacement histogram as approximately the moment when the bubble velocity counts become negligible. 


\section{Appendix B: Quantitative flowmap analysis tools}

Passage activation is studied via analysis of the measured signal intensity along transversal "cuts" perpendicular to the flow direction. We define a series of equally spaced cuts and look at how the flow is distributed throughout the cuts. We give here the quantitative process of discrimination between active and inactive passages, illustrated with images and results from the Lattice Boltzmann simulation result. The different cuts can be seen in image Figure B-1. A connected 1D line intersecting the porous area of the network along this cut is considered as a "passage". A passage can be considered active or inactive as detailed below.

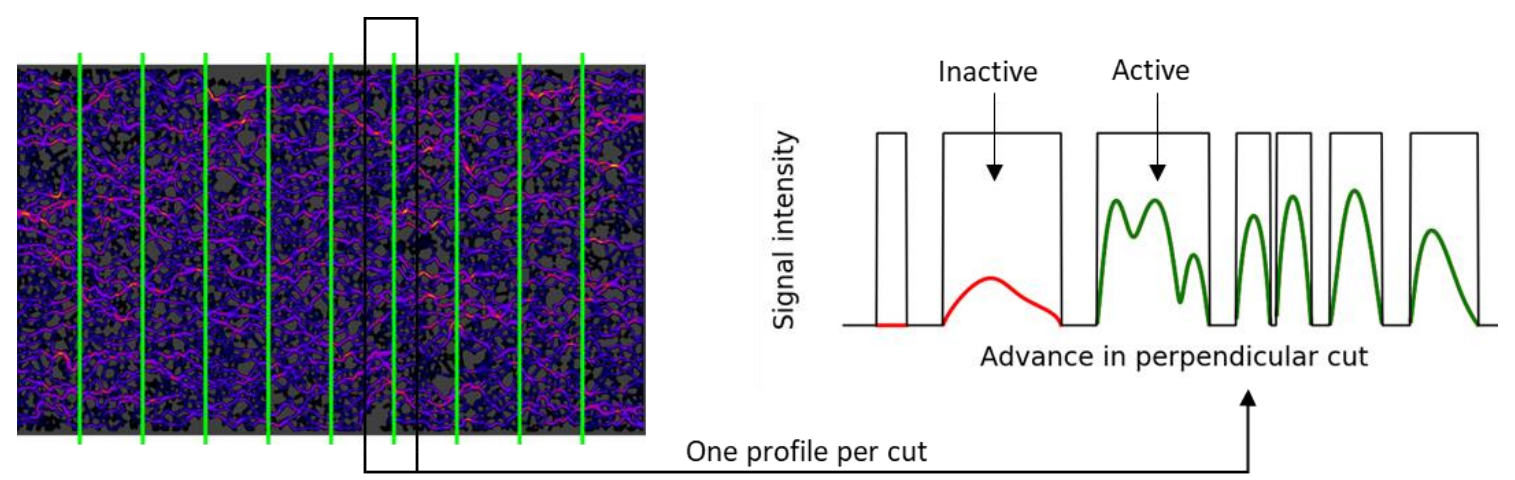

Figure B-1: Transversal model cuts from the Lattice Boltzmann simulation and measured flow signal For each cut, the total signal profile is normalized to 1 , as an intensity gradient occurs across the model as described in section XX. In each passage the summed signal can measured. The total 1D passage lengths and summed signals are tallied up for all the passages in all the cuts. A histogram is created giving the average signal for each passage length. The binning on the passage lengths is done by taking 20 points that are equidistant in logarithmic space from the largest to the smallest passage, giving 19 bins. The average signal sum per length bin serves as reference value. We observe a unimodal intensity distribution with a displacement to the right as the bin size window gets larger. The unimodal distribution indicates that there is no obvious intensity threshold to define an active/inactive passage. The method we choose is to simply take the half of the average value per bin as the cutoff value. As such, if the evaluated passage has a measured summed intensity larger than half of the reference value for its corresponding length bin, it is considered active. After each measured signal value is compared with the average value for the bin size, we can establish a passage activity ratio by dividing the number of active passages in a cut by the total number of passages in the same cut. We show in Figure B-1 a sample of the observed velocity signal bounded by the passages and colorize them according to their activity. Through this method we achieve a measurement of overall measurement of passage activation, i.e. how well is the flow distributed transversally. We can also observe the evolution of passage activation in different cuts along the model.

A supplementary global indicator of flow is produced by summing measured image intensities for each longitudinal box of the model (cutting the length of the model into 4 parallel boxes). The measured velocity signal is then summed over each box. This indicator shows longitudinal flow distribution and homogeneity. This process is shown in Figure B-2. 

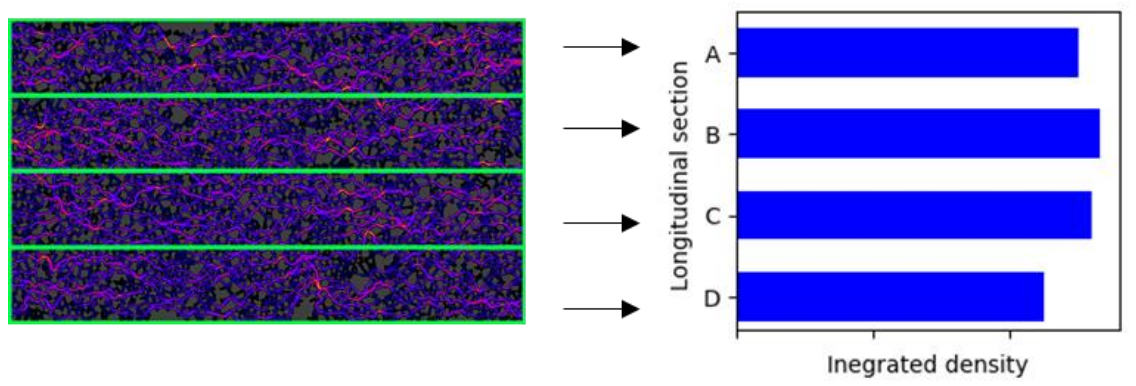

Figure B-2: Box analysis principle, flow is integrated in each longitudinal box.

\section{Appendix C: Signal intensity uniformization}

To overcome the gradient observed over the model due to bubble fragmentation and compare measured intensities fairly, we apply a multiplicative factor at each transversal column $i$ of the $N_{i} \times N_{j}$ image that corrects the intensity gradient. We explain this process here. First, the image is projected longitudinally across all the rows as to obtain a single projected intensity value $P_{i}$ for each column $\left(a N_{i} \times 1\right.$ sized vector). This vector is then smoothed to avoid overcorrecting for each local value rather than correcting for the global intensity trend. The factor $F_{i}$ is calculated at each column by $F_{i}=\max (P) / P_{i}$. Then, each row of the column $i$ is multiplied by the same value $F_{i}$ such as, in terms of intensity per column: $I_{\text {ifinal }}=$ $F_{i} \times I_{\text {iinitial }}$. In Erreur! Source du renvoi introuvable. we show the value of $F_{i}$ per column. The high number of image columns is due to the use of an upscaled image. This curve shows the difference in measured image intensity from the zone of maximal intensity measurement (the end of the model). Effectively, it gives another visualization of the bubble size evolution through the model, showing that the bubble size is relatively constant in the last third of the model, where a uniform intensity is measured.

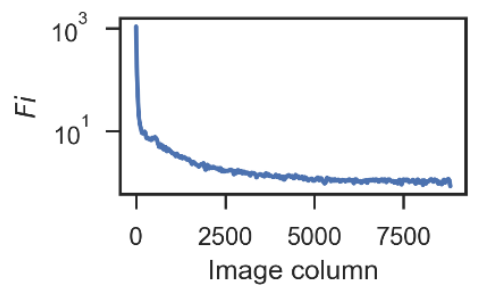

Figure C-1: Multiplicative factor for each image column in the experimental image to obtain a fair comparison of intensities per local structural element.

\section{Appendix D: Detailed local structural investigation}

We investigate parameters derived from the immediate neighborhood of the pores. The top row of Erreur ! Source du renvoi introuvable.D-1 shows contour plots of measured average intensities for each pore, against various properties of the given pore's neighborhood, such as the average, minimum and maximum pore sizes of the neighbors. In the second row, average pore intensities are shown against mean, minimal and maximal surrounding throat sizes. 

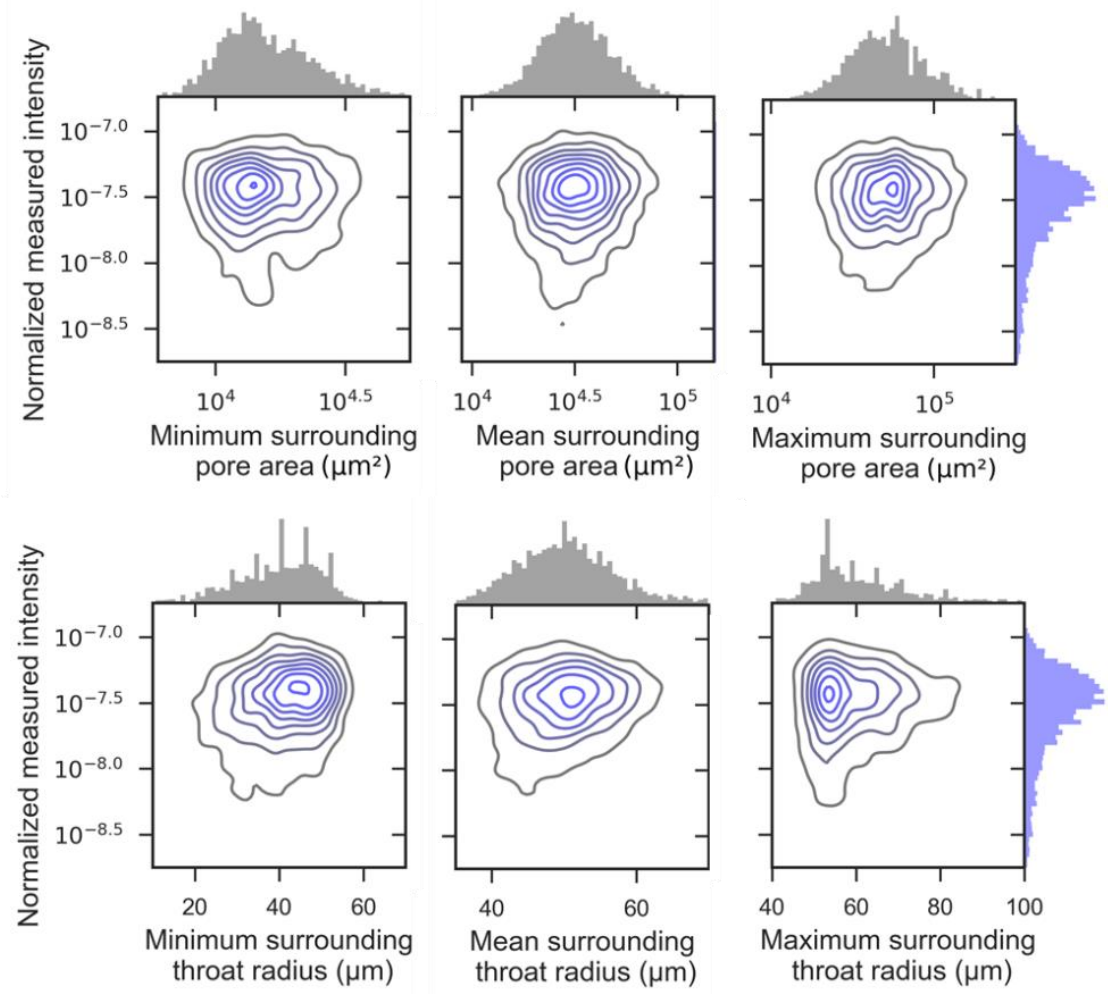

Figure D-1: Searching for relationships between local structural properties and flow intensity

We explore the specific proposition made in Géraud et al. (2016), describing preferential paths as zones with "a series of large pores are connected together". Using knowledge of the network neighborhood properties, for each pore, we create an algorithm to establish the largest possible average of connected pore sizes, with varying degree of neighbors $n$, centered on the pore we wish to evaluate. For example, if we take want to take one neighbor on each side of the pore, the algorithm will choose the two neighbors with the largest pore sizes, forming a chain of $n=3$ pores. For two neighbors on each side of the pore, the algorithm returns the largest possible pore size mean of a chain of $n=5$ connected pores ( 2 neighbors on each side plus the central pore we evaluate). We show the results for $n=3,5,7$ in Erreur ! Source du renvoi introuvable.D-2. We show that some although some positive correlation does exist, as demonstrated by the gradient of the linear fits shown as blue lines, no strong relationship was found to be evident. 

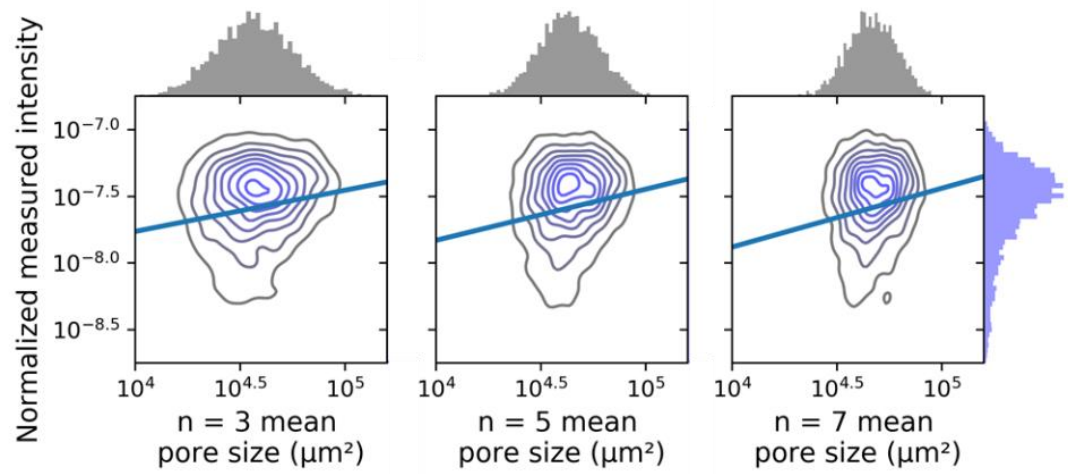

Figure D-2: Flow intensity against local neighborhood average pore size for each largest chain of n pores centered in the evaluated pore. Despite the large data dispersion, we add a linear fit to the data to show a positive relationship.

A. Ettinger, R \& J. Radke, C. (1992). Influence of Texture on Steady Foam Flow in Berea Sandstone. SPE Reservoir Engineering. 7. 83-90. 10.2118/19688-PA.

Bergeron, V.; Fagan, M. E.; Radke, C. J. (1993): Generalized entering coefficients. A criterion for foam stability against oil in porous media.In Langmuir 9 (7), pp. 1704-1713. DOI: 10.1021/la00031a017.

Conn, Charles A.; Ma, Kun; Hirasaki, George J.; Biswal, Sibani Lisa (2014): Visualizing oil displacement with foam in a microfluidic device with permeability contrast. In Lab on a chip 14 (20), pp. 3968-3977. DOI: $10.1039 / \mathrm{c} 4 \mathrm{Ic00620h}$.

Chambers, K.T., and Radke, C.J.. Micromodel foam flow study. United States: N. p., 1990. Web. doi:10.2172/6499074 Ajouter au projet Citavi par DOI.

Elka S. Basheva et al, Langmuir 2000, 16, 1000-1013

Gauteplass, Jarand; Chaudhary, Kuldeep; Kovscek, Anthony R.; Fern $\varnothing$, Martin A. (2015): Pore-level foam generation and flow for mobility control in fractured systems. In Colloids and Surfaces A:

Physicochemical and Engineering Aspects 468, pp. 184-192. DOI: 10.1016/j.colsurfa.2014.12.043.

Géraud, Baudouin; Jones, Siân A.; Cantat, Isabelle; Dollet, Benjamin; Méheust, Yves (2016): The flow of a foam in a two-dimensional porous medium. In Water Resour. Res. 52 (2), pp. 773-790. DOI:

10.1002/2015WR017936.

Géraud, Baudouin; Méheust, Yves; Cantat, Isabelle; Dollet, Benjamin (2017): Lamella Division in a Foam Flowing through a Two-Dimensional Porous Medium. A Model Fragmentation Process. In Physical review letters 118 (9), p. 98003. DOI: 10.1103/PhysRevLett.118.098003.

Haugen, Åsmund; Mani, Nima; Svenningsen, Sondre; Brattekås, Bergit; Graue, Arne; Ersland, Geir; Fern $\emptyset$, Martin A. (2014): Miscible and Immiscible Foam Injection for Mobility Control and EOR in Fractured OilWet Carbonate Rocks. In Transp Porous Med 104 (1), pp. 109-131. DOI: 10.1007/s11242-014-0323-6. 
Legland, D.; Arganda-Carreras, I. \& Andrey, P. (2016), "MorphoLibJ: integrated library and plugins for mathematical morphology with ImageJ", Bioinformatics (Oxford Univ Press) 32(22): 3532-3534

Li, Robert Feng; Yan, Wei; Liu, Shunhua; Hirasaki, George; Miller, Clarence A. (2013): Foam Mobility Control for Surfactant Enhanced Oil Recovery. In SPE Journal 15 (04), pp. 928-942. DOI: 10.2118/113910-PA.

Liontas, Rachel; Ma, Kun; Hirasaki, George J.; Biswal, Sibani Lisa (2013): Neighbor-induced bubble pinchoff. Novel mechanisms of in situ foam generation in microfluidic channels. In Soft Matter 9 (46), p. 10971. DOI: 10.1039/c3sm51605a.

Jones, S. A.; Getrouw, N.; Vincent-Bonnieu, S. (2018):Foam flow in a model porous medium. II. The effect of trapped gas.In Soft matter 14 (18), pp. 3497-3503. DOI: 10.1039/c7sm02458d.

Khatib, Z.I. \& Hirasaki, G \& Falls, A.H.. (1986). Effects of Capillary Pressure on Coalescence and Phase Mobilities in Foams Flowing Through Porous Media. SPE Reservoir Engineering. 3. 10.2118/15442-PA.

Nguyen, Quoc P.; Rossen, William R.; Zitha, Pacelli L.J.; Currie, Peter K. (2009): Determination of Gas Trapping With Foam Using X-Ray Computed Tomography and Effluent Analysis. In SPE Journal 14 (02), pp. 222-236. DOI: 10.2118/94764-PA.

Nguyen, Quoc P.; Thissen, Michiel H.G.; Zitha, Pacelli L.J.: Effect of Trapped Foam on Gas Tracer Diffusion in a Visual Microflow Model. In : SPE/DOE Improved Oil Recovery Symposium. Tulsa, Oklahoma, 200204-13.

Radke, C. J.; Gillis, J. V. (1990): A Dual Gas Tracer Technique for Determining Trapped Gas Saturation During Steady Foam Flow in Porous Media. In : SPE Annual Technical Conference and Exhibition. New Orleans, Louisiana, 1990-09-23.

Ransohoff, T. C.; Radke, C. J. (1988): Mechanisms of Foam Generation in Glass-Bead Packs. In SPE Reservoir Engineering 3 (02), pp. 573-585. DOI: 10.2118/15441-PA.

Rossen, William R. (2008): Comment on "Verification of Roof snap-off as a foam-generation mechanism in porous media at steady state". In Colloids and Surfaces A: Physicochemical and Engineering Aspects 322 (1-3), pp. 261-269. DOI: 10.1016/j.colsurfa.2008.02.034.)

Soille, Pierre; Vincent, Luc M. (1990): Determining watersheds in digital pictures via flooding simulations. In Murat Kunt (Ed.). Lausanne - DL tentative. Lausanne, Switzerland, Monday 1 October 1990: SPIE (SPIE Proceedings), pp. 240-250.

JS Tsau Reid B. Grigg (1997), Assessment of Foam Properties and Effectiveness in Mobility Reduction for CO2-Foam Floods, In SPE, International Symposium on Oilfield Chemistry

Zuiderveld, Karel (1994), "Contrast limited adaptive histogram equalization", Graphics gems IV, Academic Press ProfessioJournal Article 